\section{CDK4/6 inhibitors prolong OS}

CDK4/6 inhibitors have been proven to improve progression-free survival in women with advanced-stage hormone receptor-positive, HER2negative breast cancer, resulting in approvals of three different agents (abemaciclib, ribociclib and palbociclib), in combination with endocrine therapies, in both first-line and second-line indications. Now, two of these drugs have been shown in phase III trials to prolong overall survival (OS) in this population.

In MONARCH 2, 669 women with disease progression on prior endocrine therapy were enrolled, regardless of menopausal status, and randomly assigned (2:1) to receive fulvestrant plus either abemaciclib or placebo. Data reported at the ESMO Congress 2019 and in JAMA Oncology demonstrate a median OS of 46.7 months in the abemaciclib arm versus 37.3 months in the placebo arm (HR 0.76, 95\% CI 0.61-0.95; $P=0.01$ ). Subgroup analyses revealed that the degree of benefit from abemaciclib was generally consistent, although perhaps greater in women with highrisk disease characteristics, specifically visceral metastases (HR 0.68 , 95\% CI 0.51-0.89) and/or early resistance to endocrine therapy (HR 0.69; 95\% CI 0.45-1.04), and lower in those with bone-only disease (HR 0.91, 95\% CI 0.56-1.46).

In MONALEESA-3, 726 women were randomly allocated $(2: 1)$ to receive fulvestrant plus ribociclib or placebo; however, unlike in MONARCH 2, only postmenopausal women were enrolled, including a subset without progression on initial endocrine therapy. As also reported at the ESMO Congress 2019, OS was superior with ribociclib (median not reached versus 40 months with placebo; HR 0.72, 95\% CI 0.57-0.92; $P=0.0046)$. Notably, ribociclib provided a similar level of benefit when used in the first line (HR 0.70, 95\% CI 0.48-1.02) or second line
(HR 0.73, 95\% CI 0.53-1.00), as well as across all patient subgroups.

These results expand on the OS benefits demonstrated in premenopausal or perimenopausal women in MONALEESA-7.

In both MONARCH 2 and MONALEESA-3, CDK4/6 inhibition also increased time to second disease progression. Importantly, no new safety signals were observed in either trial.

These findings validate the currently approved indications for CDK4/6 inhibitors. Studies are now warranted to refine selection of the optimal CDK4/6 inhibitor for each patient and to overcome resistance to these agents. Predictive biomarkers to identify patients who do not benefit from CDK4/6 inhibition are also required, as emphasized by the late separation of the OS curves, and might be particularly important to the ongoing efforts to move these drugs into the adjuvant setting.

David Killock

ORIGINAL ARTICLES Sledge, G. W. et al. JAMA Oncol. https://doi.org/10.1001/jamaoncol. 2019.4782 (2019) | Slamon, D. et al. Ann. Oncol. 30 (Suppl. 5), LBA7_PR (2019)

\title{
DIAGNOSIS
}

\section{A better Al-based tool for mesothelioma}

Malignant pleural mesotheliomas (MPMs) can be classified into three histological subtypes: epithelioid, biphasic and sarcomatoid. These subtypes, however, do not fully capture the highly variable outcomes of patients with MPM. Now, MesoNet, a new classification tool now described in Nature Medicine, could enable the more accurate prediction of outcomes for these patients.

A deep-learning network was used to assign an overall survival (OS) score based on assessment of 2,048 relevant features in $\sim 10,000$ tiles of whole-slide images (WSIs) of tissue specimens from patients with MPM. This machine-learning approach was developed in training $(n=2,300)$ and testing $(n=681)$ sets and further validated in WSIs from The Cancer Genome Atlas (TCGA; $n=56$ ).

OS outcomes are typically least favourable for patients with sarcomatoid MPMs. In the test

data set, this subgroup comprised 60 patients with a median OS duration of 7.2 months. A similar median OS was observed in 60 patients in this data set predicted by MesoNet to have shorter OS, although this group included a mixture of different histologies (only $34 \%$ sarcomatoid). Patients with grade 1 epithelioid MPM typically have the most favourable OS; in the test data set, 80 such patients had a median OS of 28.1 months. The 80 patients in this data set with longer OS predicted by MesoNet had epithelioid MPMs, albeit with a mixture of grades (46\% grade 1 ).

MesoNet outperformed the predictive

these features ... might not have been noticed at diagnosis

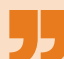
value of histology-based classification in the training ( $c$ index: 0.642 versus 0.596 ), testing ( 0.643 versus 0.598$)$ and TCGA ( 0.656 versus 0.590$)$ sets.

"Our goal was not only to focus on the performance of the model but also on its interpretability, as this is what will really provide value to the medical community," explains Charles Maussion.
The OS scores for all the individual tiles in the original data set were aggregated, and features associated with highest and lowest OS were extracted. Tiles with high OS scores usually had tubular structures and were well vascularized, whereas tiles with low OS scores mainly represented stromal features. "Some of these features were already known but others, such as inflammation, cellular diversity, vacuolization and stromal structures, had not been previously associated with poor prognosis," comments Maussion. In patients with epithelioid MPM and shorter OS, $39 \%$ of tiles displayed these features, which might not have been noticed at diagnosis.

By enabling a deeper analysis of features related with poor outcomes, this tool has the potential to improve accuracy in the diagnosis of MPM and, importantly, of other malignancies. Prospective validation of this tool is eagerly awaited.

Diana Romero

ORIGINAL ARTICLE Courtiol, P. et al. Deep learning-based classification of mesothelioma improves prediction of patient outcome. Nat. Med. 25, 1519-1525 (2019) 